ARTICLE

\title{
Fast-field-cycling ultralow-field nuclear magnetic relaxation dispersion
}

\author{
Sven Bodenstedt ${ }^{1}$, Morgan W. Mitchell ${ }^{1,2} \&$ Michael C. D. Tayler (iD) ${ }^{1 凶}$
}

Optically pumped magnetometers (OPMs) based on alkali-atom vapors are ultra-sensitive devices for dc and low-frequency ac magnetic measurements. Here, in combination with fast-field-cycling hardware and high-resolution spectroscopic detection, we demonstrate applicability of OPMs in quantifying nuclear magnetic relaxation phenomena. Relaxation rate dispersion across the $\mathrm{nT}$ to $\mathrm{mT}$ field range enables quantitative investigation of extremely slow molecular motion correlations in the liquid state, with time constants $>1 \mathrm{~ms}$, and insight into the corresponding relaxation mechanisms. The $10-20 \mathrm{fT} / \sqrt{\mathrm{Hz}}$ sensitivity of an OPM between $10 \mathrm{~Hz}$ and $5.5 \mathrm{kHz}{ }^{1} \mathrm{H}$ Larmor frequency suffices to detect magnetic resonance signals from $0.1 \mathrm{~mL}$ liquid volumes imbibed in simple mesoporous materials, or inside metal tubing, following nuclear spin prepolarization adjacent to the OPM. High-resolution spectroscopic detection can resolve inter-nucleus spin-spin couplings, further widening the scope of application to chemical systems. Expected limits of the technique regarding measurement of relaxation rates above $100 \mathrm{~s}^{-1}$ are discussed.

\footnotetext{
${ }^{1}$ ICFO - Institut de Ciències Fotòniques, The Barcelona Institute of Science and Technology, Castelldefels (Barcelona), Spain. ${ }^{2}$ ICREA - Institució Catalana de Recerca i Estudis Avançats, Barcelona, Spain. ${ }_{\text {email: michael.tayler@icfo.eu }}$
} 
$\mathrm{N}$ ano-scale dynamic processes that occur on ms to $\mu$ s time scales, such as protein folding, aqueous complexation, and surface adsorption phenomena, are often probed using nuclear magnetic relaxation dispersion (NMRD) techniques ${ }^{1-3}$, in which field-dependent relaxation rates of nuclear spins are used to infer correlation times for molecular reorientation ${ }^{4,5}$ and diffusive transport. Beyond fundamental interests, insights from NMRD such as surface fractal dimension and roughness provide models for industrial catalysis and petrology, where liquids are confined inside porous solids and molecular diffusion is restricted by surface geometry ${ }^{6}$ as well as adsorption ${ }^{7}$, and in medicine assist the design of molecular agents for relaxation-contrast magnetic resonance imaging $(\mathrm{MRI})^{8}$. Furthermore, if coupled with spectroscopic dispersion via chemical shifts or spin-spin couplings, the dynamics can be related to specific molecular functional groups, facilitating analyses of chemical mixtures and biological specimens ${ }^{9}$.

Accurate correlation times $\tau_{\mathrm{c}}$ can be obtained by measuring nuclear spin relaxation across a range of Larmor frequencies $B \gamma_{I} \ll \tau_{\mathrm{c}}^{-1}$ to $B \gamma_{I} \gg \tau_{\mathrm{c}}^{-1}$, where $B$ is the field strength and $\gamma_{I}$ is the nuclear gyromagnetic ratio. Extremely slow correlations thus require measurements at ultralow magnetic fields within shielded enclosures such as a MuMetal chamber. The main existing NMRD technique uses fast-field-cycling (FFC) electromagnets $^{10-13}$ of around $1 \mathrm{~T}$ for efficient inductive NMR signal detection, but these must be used unshielded with active cancelation of ambient fields to access below the geomagnetic field range ${ }^{14,15}$. Alternatively, NMRD is performed by transporting samples between persistent high- and ultralow-field locations ${ }^{16-20}$, but relatively slow transport times limit the observable $\tau_{\mathrm{c}}$ at the high end. The limits of these existing techniques are illustrated by the magenta- and blue-shaded regions, respectively, of Fig. 1.

In this work, we introduce a third scenario to address the topleft portion of Fig. 1 that lies outside the reach of inductive NMR pickup. The speed of the FFC approach is combined with the lowfrequency sensitivity of a spin-exchange-relaxation-free $(\mathrm{SERF})^{21-28}$ optically pumped magnetometer (OPM) to perform NMRD at ${ }^{1} \mathrm{H}$ Larmor frequencies from $1 \mathrm{~Hz}$ to $10 \mathrm{kHz}$, corresponding to the region of Fig. 1 shaded in green. The high sensitivity of SERF OPMs of order $1 \mathrm{fT} / \sqrt{\mathrm{Hz}}^{29,30}$ at signal

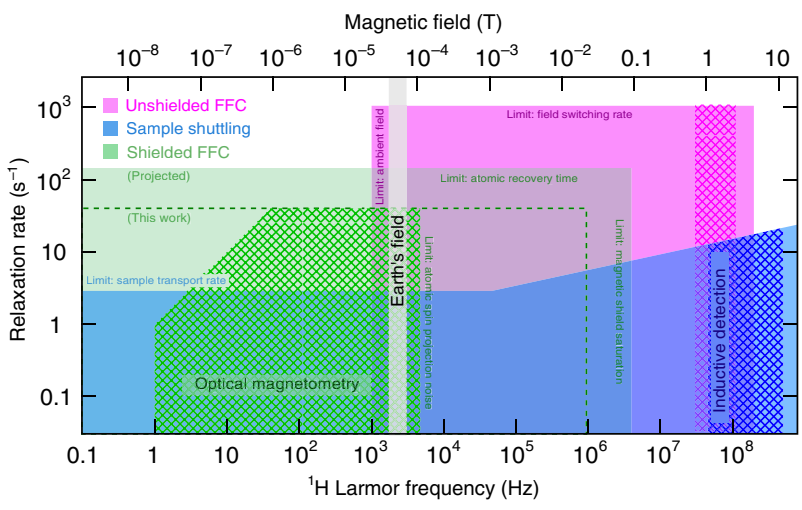

Fig. 1 Limits of existing NMRD techniques. Boundaries of the colored zones in the horizontal dimension indicate upper limits of longitudinal relaxation rate, $\left(T_{1, l}^{-1} \mathrm{~s}^{-1}\right)$, imposed by signal receiver dead time and/or speed of field-switching events. Boundaries in the vertical dimension indicate limits to the range of magnetic fields achievable by electromagnetic sources. Cross-hatching indicates Larmor frequencies where NMR signals are detected and corresponds to measurable transverse relaxation rates $T_{2,1}^{-1}\left(\mathrm{~s}^{-1}\right)$. The diagonal of slope 1 corresponds to the spectroscopic resolution limit where rates of Larmor precession and transverse relaxation are equal: $B \gamma_{l} /(2 \pi)=T_{2, l}^{-1} \sim T_{1, l}^{-1}$. frequencies down to a few $\mathrm{Hz}$, rivals the best superconducting quantum interference device (SQUID) $)^{31,32}$ and high- $Q$ inductivepickup magnetometers below Earth's field ${ }^{33,34}$, with the advantage of cryogen-free operation and simple tuning based on Hartmann-Hahn matching of the OPM and NMR spin ensembles. In the ultralow-field NMRD context, the OPM is compatible both with MuMetal shielding and relatively weak prepolarizing fields of order $10 \mathrm{mT}$. Magnetic fields for relaxation and detection are supplied accurately and precisely (within $1 \mathrm{nT}$ ) following a one-off calibration procedure and can be cycled in less than $1 \mathrm{~ms}$, resulting in atomic-response-limited dead times. Based on this configuration, we are able to study spin relaxation phenomena that cannot be probed using conventional inductive field-cycling NMR procedures: (1) relaxation of liquids encased in metal tubing, demonstrated using aqueous solutions of paramagnetic impurities; (2) the full frequency dependence for motional correlations in a system of $n$-octane $\left(n-\mathrm{C}_{8} \mathrm{H}_{18}\right)$ and $n$-decane $(n$ $\mathrm{C}_{10} \mathrm{H}_{22}$ ) absorbed in nanoscale confinement upon porous alumina and titania, as an example relevant to research in catalysis. Results unambiguously support dynamics models involving molecular diffusion among paramagnetic sites on the pore surface; (3) chemical species resolution via spin-spin J couplings.

\section{Results}

Dynamics from NMRD. When it covers the appropriate range of fields and time scales, the relaxation measured in NMRD can relate model parameters of interest to molecular motion, surface structure, and molecule-surface interactions ${ }^{35-37}$. A central quantity of interest is the time correlation function $g(\tau)=\langle x(t) x(t+\tau)\rangle /\langle x(t) x(t)\rangle$ of the molecular motion. This is related to observable relaxation by the quantity $j(\omega)=\int_{0}^{\infty} g(\tau) \cos (\omega \tau) \mathrm{d} \tau$, i.e. the cosine transform of $g(\tau)$. Here we assume a simple but useful model where the local field is inhomogeneous, with a randomly oriented component of rootmean-square amplitude $B_{\text {rms }}$. The longitudinal relaxation rate is $\left[T_{1, I}\left(\omega_{I}\right)\right]^{-1}=\gamma_{I}^{2} B_{\mathrm{rms}}^{2} j\left(\omega_{I}\right)$ under standard perturbation (i.e. Redfield $\left.{ }^{35}\right)$ assumptions. In the ideal case of unrestricted diffusion, a single correlation time is found, where $g(\tau) \propto \exp \left[-\left(\tau / \tau_{\mathrm{c}}\right)\right]$ and the spectral density is a Lorentzian: $j(\omega)=\tau_{c} /\left(1+\omega^{2} \tau_{c}^{2}\right)$, where $\tau_{\mathrm{c}}$ is the characteristic diffusion time. Inverse-square power-law behavior is thus expected for $T_{1, I}^{-1}$ vs. $\omega_{I}$, for $\omega_{I} \gg \tau_{\mathrm{c}}^{-1}$.

Scenarios of constrained Brownian motion ${ }^{38}$ such as diffusion in pores may yield several concurrent dynamics modes. Fitting to a distribution of correlation times may be more appropriate: $\left[T_{1, I}\left(\omega_{I}\right)\right]^{-1}=\gamma_{I}^{2} \int_{0}^{\infty} B_{\mathrm{rms}}^{2} p\left(\tau_{\mathrm{c}}\right) \tau_{\mathrm{c}} /\left(1+\omega_{I}^{2} \tau_{\mathrm{c}}^{2}\right) \mathrm{d} \tau_{\mathrm{c}}{ }^{39,40}$, where $p\left(\tau_{\mathrm{c}}\right)$ represents a probability distribution normalized to $\int_{0}^{\infty} p\left(\tau_{c}\right) \mathrm{d} \tau_{\mathrm{c}}=1$. Kimmich and co-workers examine this approach to explain power-law relaxation behavior in porous glasses: $T_{1, I} \propto \omega_{I}^{\xi}$, where $0<\xi<2^{41,42}$. Surface-induced relaxation is attributed to "molecular reorientation mediated by translational displacement" (RMTD), where diffusion across a rugged pore surface modulates intra-molecular spin-spin dipolar couplings and $p\left(\tau_{c}\right)$ is linked to the surface fractal dimension. A breakdown of the power law at low frequencies $\omega_{I}$ indicates a maximum $\tau_{c}$, which is connected to the longest distance a molecule can diffuse before leaving the surface phase or experiencing a different surface structure. The value should depend on the molecule, due to different diffusion coefficients, as well as the porous medium.

Moreover towards zero Larmor frequency, $T_{1, I}$ tends to a plateau defined by $\left[T_{1, I}(0)\right]^{-1}=\gamma_{I}^{2} \int_{0}^{\infty} B_{\mathrm{rms}}^{2} p\left(\tau_{\mathrm{c}}\right) \tau_{\mathrm{c}}=\gamma_{I}^{2} B_{\mathrm{rms}}^{2}\left\langle\tau_{c}\right\rangle$, where $\left\langle\tau_{c}\right\rangle$ is the mean correlation time ${ }^{37}$. This and the above measures all require $T_{1, I}$ to be known for frequencies below $\tau_{c, \text { max }}^{-1}$, motivating the ultralow-field measurement capability. 

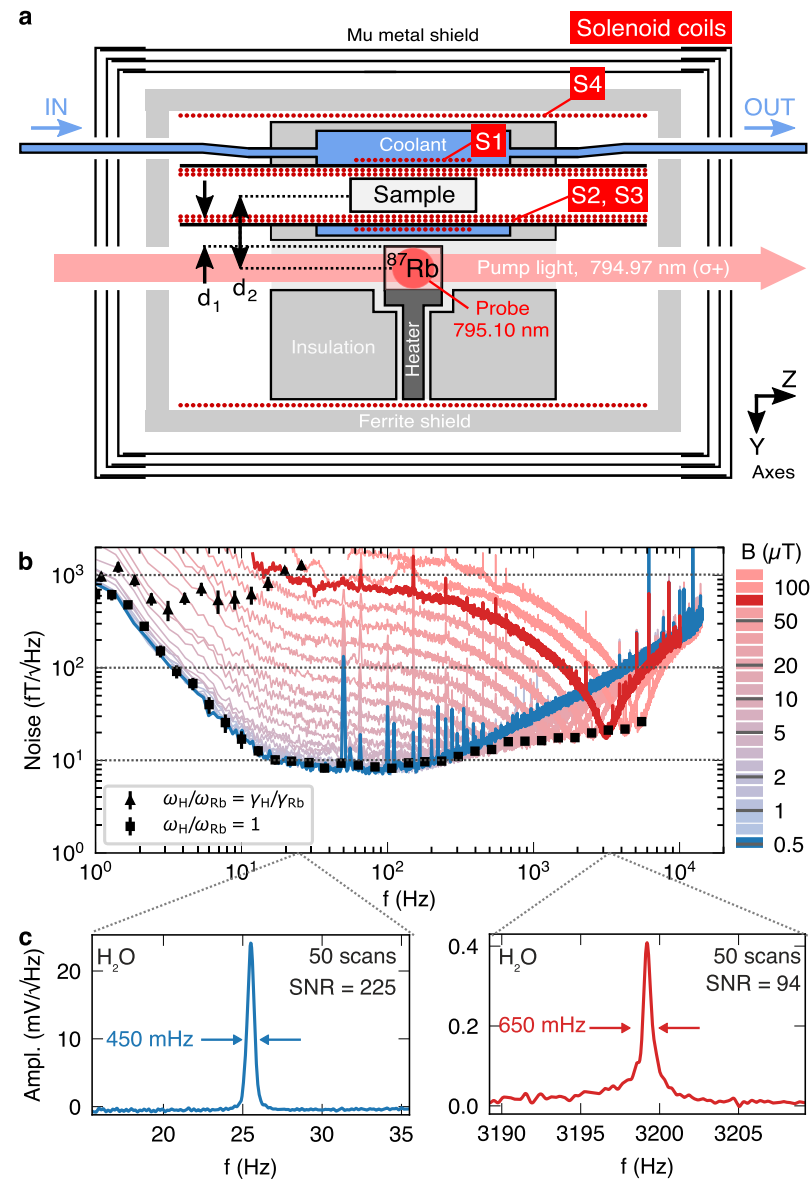

Fig. 2 Tunable NMR detection via optically pumped magnetometer. a Schematic view of the apparatus through a vertical cross section. Standoff distance between sample container atomic vapor cell $d_{1}=3.5 \mathrm{~mm}$. Centerto-center distance between atomic cell and NMR sample $d_{2}=10 \mathrm{~mm}$. $\mathbf{b}$ Frequency dependence of magnetometer noise in field-equivalent units at bias fields $B_{z, l}$ from 0.1 to $100 \mu \mathrm{T}$. Plot markers indicate sensitivity at the ${ }^{1} \mathrm{H}$ Larmor frequency under the conditions of $(\boldsymbol{\square})$ tuned and $(\boldsymbol{\Delta})$ untuned ${ }^{87} \mathrm{Rb}$ Larmor frequencies; error bars correspond to the sensitivity's root-meansquare deviation within a $1 \mathrm{~Hz}$ window around the Larmor frequency. c Representative NMR signal and noise amplitudes in the tuned case for a sample of $1.8 \mathrm{~mL}$ milli-Q water, measured after pre-polarization at $20 \mathrm{mT}$ and a transverse $\pi / 2$ pulse.

Tunable NMR detection using optical magnetometry. Figure 2a shows an experimental setup for FFC NMR with OPM detection. A $2 \mathrm{~mL}$ vial containing the NMR sample sits within four coaxial solenoids (S1-S4), which provide $z$-oriented fields. Liquid coolant flows around the sample chamber, with S1 moreover immersed in the flowing coolant to maintain a sample temperature around $30{ }^{\circ} \mathrm{C}$. The short $\mathrm{S} 1$ solenoid is used to produce fields up to $20 \mathrm{mT}$ to polarize the nuclear spins in the sample, while S2 and S3 provide weaker fields for Larmor precession. Adjacent to this chamber is a heated glass cell containing ${ }^{87} \mathrm{Rb}$ vapor, with optical access along $x$ and $z$ directions for probing and pumping of the alkali spin angular momentum $\mathbf{S}$, respectively.

A magnetic field $\mathbf{B}=\left(B_{1} \cos \omega t, B_{1} \sin \omega t, B_{z, S}\right)$ in Cartesian coordinates is assumed at the atoms, as the sum of a constant bias field $B_{z, \mathrm{~S}}$ along the $z$-axis and an effective rotating field $B_{1}$ induced by the precession of nuclear magnetization in the NMR sample in the $x y$ plane. Assuming the nuclei experience a field $B_{z, \mathrm{I}}$ along the $z$-axis, then $\omega=\omega_{I}=\gamma_{I} B_{z, \mathrm{I}}$, and $B_{1}$ is proportional to the amplitude of the nuclear magnetization.
Dynamics of $\mathbf{S}$ are adequately described in the SERF regime by a polarization vector model where the $x$-axis component of $\mathbf{S}$ under steady-state pump-probe and a transverse rf field of angular frequency $\omega$ is given by ${ }^{30}$

$$
S_{x}=\frac{g_{S} R_{\mathrm{op}} T_{2, S}^{2}}{2 q^{2}}\left[\frac{\cos \omega t+\left(\omega-\omega_{S}\right) T_{2, S} \sin \omega t}{1+\left(\omega-\omega_{S}\right)^{2} T_{2, S}^{2}}\right] B_{1} .
$$

Here, $\omega_{\mathrm{S}}=g_{\mathrm{S}} B_{z, \mathrm{~S}} / q$ is the Larmor frequency, $g_{\mathrm{S}}$ is the gyromagnetic ratio, $q$ is the nuclear slowing down factor, $R_{\mathrm{op}}$ is the optical pumping rate and $T_{2, S}^{-1}$ is the transverse relaxation rate of the alkali atom ensemble. According to the above Eq. (1), the atomic response to $B_{1}$ is strongest for matched precession frequencies of the spin species: $\omega_{\mathrm{I}}=\omega_{\mathrm{S}}$. Thus the OPM is tunable to a given NMR frequency $\omega_{I}$ by setting the magnetic field at the atoms to $B_{z, \mathrm{~S}}= \pm\left(q / g_{s}\right) \omega_{I}= \pm \gamma_{I}\left(q / g_{s}\right) B_{z, \mathrm{I}}$. This adjustment is permitted since $B_{z, \mathrm{I}}$ is the superposition of the fields in the interior of coils $\mathrm{S} 2+\mathrm{S} 3+\mathrm{S} 4$, while $B_{z, \mathrm{~S}}$ is the superposition of fields from $\mathrm{S} 4$ and the much weaker exterior field of $\mathrm{S} 2+\mathrm{S} 3$.

For Larmor frequencies $\omega_{I} /(2 \pi)$ between 10 and $200 \mathrm{~Hz}$ the magnetometer noise is below $10 \mathrm{fT} / \sqrt{\mathrm{Hz}}$ (Fig. 2b), limited by noise in the lasers and to a lesser extent the Johnson noise of the coils $\mathrm{S} 1+\mathrm{S} 2+\mathrm{S} 3$. The spin projection noise estimated from the atom density $n_{S} \approx 10^{20} \mathrm{~m}^{-3}$, temperature $150{ }^{\circ} \mathrm{C}$ and coherence time $T_{2, \mathrm{~S}} \approx 3 \mathrm{~ms}$ is $\sqrt{n_{S} g_{S}^{2} T_{2, S} / q} \sim 1.1 \mathrm{fT} / \sqrt{\mathrm{Hz}}$. Above fields $B_{z, \mathrm{~S}} \approx 100 \mathrm{nT}, \omega_{\mathrm{S}}$ starts to become comparable to $1 / T_{2, \mathrm{~S}}$, marking the limit of the SERF regime, and the magnetometer noise rises above $20 \mathrm{fT} / \sqrt{\mathrm{Hz}}$. Overall, as Fig. 2c illustrates, NMR signals are obtainable at fields where Larmor frequencies are around 100 times higher than the atomic bandwidth. In contrast, without tuning, the combined atomic and nuclear spin system yields a relatively narrow operating range for NMR, quantified by the half-width at half-height of Equation (1): $\Delta \omega_{\mathrm{I}} /(2 \pi) \approx g_{\mathrm{S}} T_{2, \mathrm{~S}} /$ $(2 \pi q) \approx 80 \mathrm{~Hz}$.

Dissolved paramagnetic species in liquids. Many singlecomponent liquids and simple solutions are characterized by an exponential correlation function for molecular tumbling, with a time constant $\tau_{\mathrm{c}}$ in the low ps range. Unless much slower additional motion processes exist the NMR relaxation times $T_{1, I}$ and $T_{2, \mathrm{I}}$ are independent of magnetic field below $\left.B_{z, \mathrm{I}} \ll\left(\gamma_{I} \tau_{\mathrm{c}}\right)^{-1}\right) \sim$ $0.1 \mathrm{~T}$, all the way down to ultralow field.

Here we observe the dependence of relaxation in aqueous solutions of the paramagnetic compound 4-hydroxy-2,2,6,6-tetramethylpiperidin-1-oxyl (TEMPOL). TEMPOL is a chemical oxidant under study elsewhere for potential therapeutic properties ${ }^{43}$, as well as a source of nuclear spin hyperpolarization ${ }^{44,45}$ that can achieve enhanced sensitivity in NMR. Sequences A and B are used, respectively, to measure ${ }^{1} \mathrm{H} T_{1, I}$ and $T_{2, I}$ (Fig. 3a).

In sequence $\mathrm{A}$, nuclear spin prepolarization at $20 \mathrm{mT}$ is followed by switching to a lower magnetic field for a time $\tau_{1}$, before a dc $\pi / 2$ pulse induces NMR-free nuclear precession about the $z$-axis. The amplitudes of the NMR signal, $s_{\mathrm{A}}$, are fit well by the function $s_{A} \propto \exp \left(-\tau_{1} / T_{1, I}\right)$ and the observed relaxation rates scale linearly with concentration of the paramagnetic dopant as $T_{1, I}^{-1}=\left(T_{1, I}^{(0)}\right)^{-1}+k_{1}[\mathrm{TEMPOL}]$, where $T_{1, I}^{(0)}$ is the relaxation time at zero solute (Fig. $3 \mathrm{~b}$ ). The relaxivity parameter $k_{1}=$ $0.453(5) \mathrm{s}^{-1} \mathrm{mmol}^{-1} \mathrm{dm}^{3}$ is in good agreement with literature values at the high-field end 44,45 , which gives confidence in the method. In sequence $B$, the initial $\pi / 2$ pulse is followed by a Hahn-echo to refocus transverse magnetization after time $\tau_{2}$. Signal amplitudes are fit well by the expected function $\exp \left(-\tau_{1} / T_{1, I}-\tau_{2} / T_{2, I}\right)$ and provide a transverse relaxivity 
a Sequence
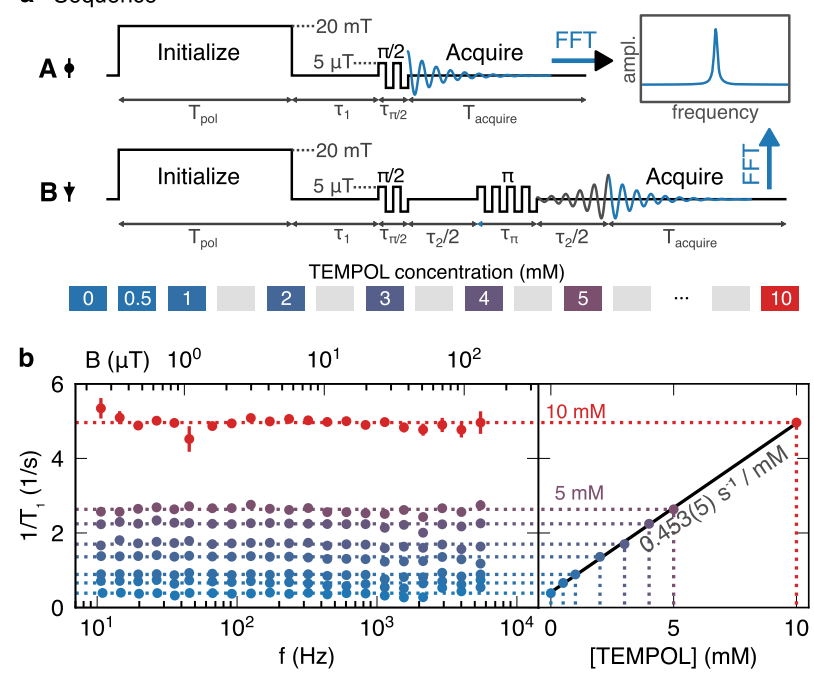

c
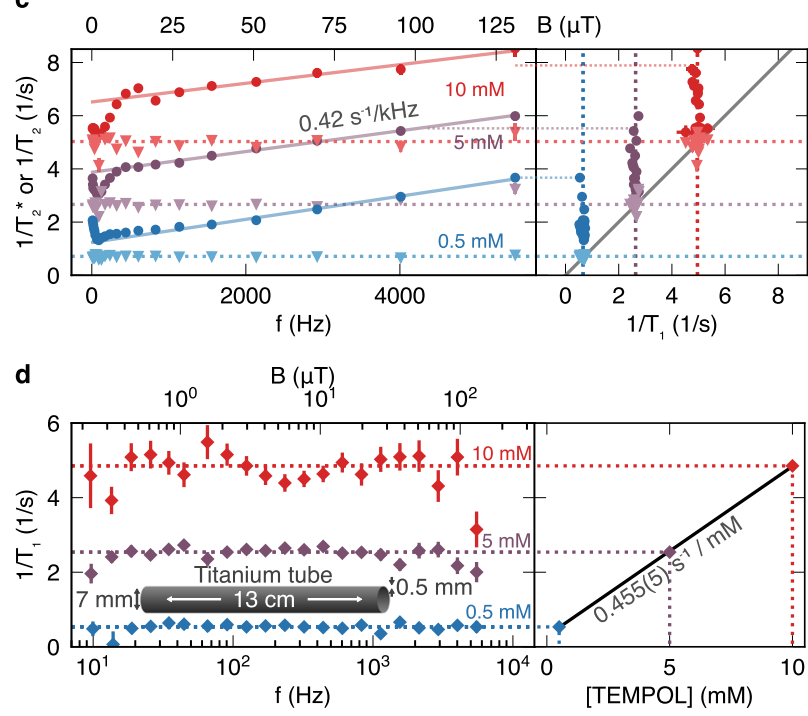

Fig. 3 Ultralow-field NMR relaxation of $\boldsymbol{I}={ }^{\mathbf{1}} \mathbf{H}$ in aqueous TEMPOL solutions at $30^{\circ} \mathrm{C}$. a Sequences $A$ and $B$ measure longitudinal $\left(T_{1, l}^{-1}\right.$; diamond plot markers) and transverse $\left(T_{2,1}^{-1}\right.$ and $\left(T_{2,1}^{*}\right)^{-1}$; triangle and circle plot markers) relaxation rates. Vertical axis (not to scale) shows field strength with the polarization field along $z$ and the $\pi / 2$ and $\pi$ pulses along $y$. b Field dependence of rates $T_{1, l}^{-1}$ across $25 \mathrm{nT}<B_{z, l}<130 \mu \mathrm{T}$, $1.8 \mathrm{~mL}$ sample volume. Rates are linearly proportional to TEMPOL concentration. c Field dependence of $\left(T_{2,1}^{*}\right)^{-1}$ and $\left(T_{2,1}\right)^{-1}$. $\left(T_{2,1}^{*}\right)^{-1}$ depends weakly on field due to instrument-specific gradients in $B_{z, l}$ and $B_{z, S}$, while $T_{2,1}^{-1} \approx T_{1,1}^{-1}$. d Relaxation rates for TEMPOL solutions encased in $0.5 \mathrm{~mm}$-thick titanium tube, $0.1 \mathrm{~mL}$ sample volume. All vertical error bars represent estimated standard deviation errors obtained from least-squares regression analysis.

parameter $k_{2}=0.455(5) \mathrm{s}^{-1} \mathrm{mmol}^{-1} \mathrm{dm}^{-3} \quad$ defined by $T_{2, I}^{-1}\left(B_{z, \mathrm{I}}\right.$, $[$ TEMPOL $\left.]\right)=T_{2, \mathrm{I}}^{-1}\left(B_{z, \mathrm{I}}, 0\right)+k_{2}\left(B_{z, \mathrm{I}}\right)[$ TEMPOL $]($ Fig. $3 \mathrm{c}$, triangle plot markers). The result $k_{2}=k_{1}$ holds down to $\mathrm{nT}$ fields, which confirms isotropic molecular tumbling in the fast motion limit and absence of slow motional correlations.

The transverse decay rates are also well approximated by $\left(T_{2, I}^{*}\right)^{-1}=\mathrm{FWHM} /(2 \pi)$ obtained from line widths in the Fouriertransform NMR spectra of sequence $A$, due to relatively low inhomogeneity in $B_{z, I}$. Here, $\mathrm{S} 2+\mathrm{S} 3$ produce field gradients $\mathrm{d} B_{z}$, ${ }_{I} / \mathrm{d} z$ and smaller components along $x$ and $y$ due to tilt imperfections in the coil windings, resulting in a linear dependence $\left(\mathrm{d} / \mathrm{d} B_{z, \mathrm{I}}\right)\left(T_{2, \mathrm{I}}^{*}\right)^{-1}=0.01 \mathrm{~s}^{-1} \mu \mathrm{T}^{-1}$ (or 4 ppk of $\left.B_{z, \mathrm{I}}\right)$ observed above $500 \mathrm{~Hz}{ }^{1} \mathrm{H}$ frequency. We also note that $\mathrm{S} 4$ is centered on the ${ }^{87} \mathrm{Rb}$ cell and not on the NMR sample, therefore gradients may cancel out at some Larmor frequencies; this effect is attributed to the line narrowing at around $200 \mathrm{~Hz}$. Overall the results show that the NMR linewidth stays below $1 \mathrm{~Hz}$ even up to geomagnetic fields, and that TEMPOL causes no further detriment to spectroscopic resolution in the zero/ultralow-field range.

To further demonstrate the application potential of the technique we highlight that ultralow-field cycling and NMR detection is compatible with metal sample enclosures. NMR signals can be detected without amplitude loss up to $\mathrm{kHz}$ Larmor frequencies when $0.1 \mathrm{~mL}$ aliquots of the TEMPOL solutions are contained inside a titanium alloy tube (outer diameter $8 \mathrm{~mm}$, inner diameter $7 \mathrm{~mm}$, pressure rating $13 \mathrm{MPa}$ ). Relaxation rates $1 / T_{1, I}$ for samples with and without the metal tube, shown in Fig. 3d, are identical within measurement error to those of Fig. 3b. Larger error bars are due to the smaller sample volume giving lower signal to noise. This measurement is impossible via conventional fast-field cycling NMR techniques, where eddy currents in metal strongly attenuate the amplitude of highfrequency NMR signal and also limit the rate of field switching. Transverse relaxation rates are also unaffected by the presence of the metal tube, from which we may conclude that eddy currents are negligible over the relatively small $(\mathrm{mT})$ range of field switching. The approach may therefore open the way to study relaxation in unexplored contexts, for instance high-pressure fluids (e.g. supercritical fluids), flow in pipes, foil-sealed products (e.g. foods, pharmaceuticals), and (e.g. lead-, tungsten-) sealed radioactive samples.

Liquids confined in porous materials. To demonstrate insight into molecular motion near pore surfaces we study the ${ }^{1} \mathrm{H}$ spin relaxation of n-alkane hydrocarbons confined within matrices of alumina ( $\gamma$ polymorph, $9 \mathrm{~nm}$ mean pore diameter) and titania (anatase polymorph, 7-10 $\mathrm{nm}$ mean pore diameter). These simple inorganic oxides in their mesoporous form possess catalytic features due to their high specific surface area, Lewis acidic sites, and option of chemical treatments including metalization to activate the pore surface. Yet, owing to the frequency range of conventional NMRD techniques, there is limited understanding of how molecular dynamics and surface site properties relate to long- $\tau_{\mathrm{c}}$ relaxation processes, even without surface functionalization ${ }^{11}$.

Figure 4a shows ${ }^{1} \mathrm{H}$ relaxation rates at $30^{\circ} \mathrm{C}$ for imbibed $n$ alkanes, measured between $1 \mathrm{~Hz}$ and $5.5 \mathrm{kHz}$ Larmor frequency using the sequence shown in Fig. 4b. Due to excess noise in the magnetometer below $100 \mathrm{~Hz}$ (including mains electricity noise and $1 / f$ noise, see Fig. $4 c$ ), fast field switching between relaxation and detection events is the preferred measurement option to probe the lowest fields, where the NMR signal is always detected at frequency above $100 \mathrm{~Hz}$. Above $100 \mathrm{~Hz}$ Larmor frequency, the noise floor is low enough to detect NMR signals at the relaxation field, without switching. The measurable NMR relaxation is limited in principle to rates $T_{1, I}^{-1}<R_{\mathrm{op}}^{-1}$, where the latter is of order $300 \mathrm{~s}^{-1}$. However, in practice, the limit is $T_{1, I}^{-1}<T_{2, S}^{-1}$ or around $100 \mathrm{~s}^{-1}$ since the atomic precession signal causes a $10 \mathrm{~ms}$ dead time caused following the $\pi / 2$ pulse (see Fig. $4 d$ ).

The main feature of Fig. $4 \mathrm{a}$ is the weak dispersion in $T_{1, I}^{-1}$ for each alkane, and moreover between the two porous materials, across the conventional FFC-NMR frequency range $10 \mathrm{kHz}$ to 1 $\mathrm{MHz}^{11}$. The relaxation rate for each alkane depends only slightly on the porous material, therefore bulk effects dominate the relaxation process in this range. In contrast, relaxation rates below $10 \mathrm{kHz}$ depend strongly on the material and mechanisms related to the surface are prominent, with the higher values being observed towards zero field. The $T_{1, I}$ dispersion in titania is much 


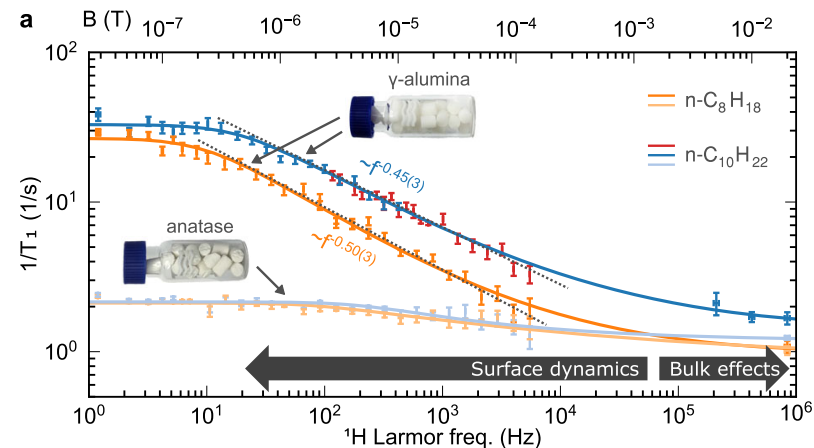

b
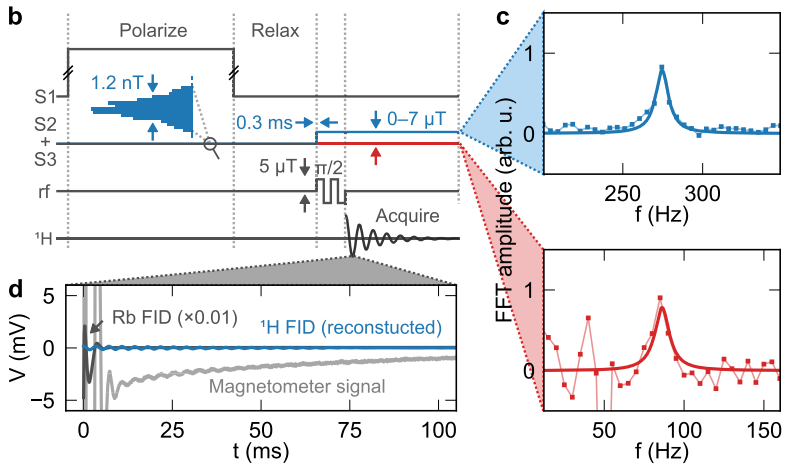

Fig. 4 Ultralow-field FFC NMR of pore-confined fluids. a Magnetization decay rates for $n$-octane and $n$-decane in porous $\gamma$-alumina and anatase titania. For $n$-decane in $\gamma$-alumina, colors indicate the NMR detection field, as discussed in the main text and represented in the rest of the figure. Data above $100 \mathrm{kHz}$ correspond to magnetization buildup rates in the S1 field; vertical error bars represent estimated standard deviation errors obtained from least-squares regression analysis. $\mathbf{b} \mathrm{FFC}$ sequence used to measure $T_{1,1}$ decay at ultralow field; c frequency- and $\mathbf{d}$ time-domain NMR signal of $n$ decane in $\gamma$-alumina after relaxation at $\omega_{l} /(2 \pi)=85 \mathrm{~Hz}$. In $\mathbf{c}$, the NMR signal-to-noise ratios (snr) illustrate the FFC requirement below $100 \mathrm{~Hz}$ : poor snr at $\omega_{1} /(2 \pi)=85 \mathrm{~Hz}$ is due to $1 / f$ and $50 \mathrm{~Hz}$ noise of the OPM; FFC switching to $\omega_{1} /(2 \pi)=275 \mathrm{~Hz}$ between relaxation and detection events is optimal for high snr and short dead time. The blue and red color coding also serves to indicate the method used to measure $T_{1, l}$ for $n$-decane in $\gamma$ alumina, in part (a) of the figure. The reconstructed time-domain signal in (d, blue curve) equates to the Lorentzian line shape fitted in (c).

weaker than in $\gamma$-alumina; $T_{1, I}^{-1}$ reaches only around $2 \mathrm{~s}^{-1}$ below $200 \mathrm{~Hz}$, compared to $30 \mathrm{~s}^{-1}$ for alumina. Although the two materials have similar mean pore diameter and surface area/ volume ratio, surface-induced relaxation is not so active in the first material. It is known from electron spin resonance spectroscopy ${ }^{46}$ that the alumina contains a higher concentration of paramagnetic impurity- $\left[\mathrm{Fe}^{3+}\right] \approx 2 \times 10^{16} \mathrm{~g}^{-1}$ (i.e., ions per unit mass of the dry porous material) in alumina vs. $2 \times 10^{15} \mathrm{~g}^{-1}$ in titania-suggesting that the lower-frequency relaxation mechanism involves dipole-dipole coupling between ${ }^{1} \mathrm{H}$ and the surface spins, rather than surface-induced modulation of intra-molecular ${ }^{1} \mathrm{H}-{ }^{1} \mathrm{H}$ spin couplings.

Between $\omega_{I} /(2 \pi)=50$ and $5000 \mathrm{~Hz}$, the longitudinal relaxation in $\gamma$-alumina obeys a power-law frequency dependence: $T_{1, I} \propto \omega_{I}^{\xi}$. Fitted slopes $-\mathrm{d}\left(\log T_{1, I}^{-1}\right) / \mathrm{d}\left(\log \omega_{I}\right)$ give exponents $\xi=0.50 \pm 0.03$ for octane and $\xi=0.45 \pm 0.03$ for decane. Such values are consistent with simple numerical simulations in which imbibed molecules randomly walk within a dilute matrix of nonmobile spins-such as surface paramagnets - where the strength of dipole-dipole interactions between the two spin species scales with the inverse cube of their instantaneous separation ${ }^{39}$. This nonlinear dependence results in an example of Lévy walk statistics. A detailed characterization of these effects in the alumina system is ongoing work.

Although developing an analytical model for the surface dynamics is outside the scope of this paper, for analysis of the correlation time it suffices to fit the measured relaxation rates by a stretched Lorentzian function $\left[T_{1, I f \text { fit }}\left(\omega_{I}\right)\right]^{-1}=\left[T_{1, I}(0)\right]^{-1} /\left(1+\tau_{c}^{2} \omega_{I}^{2}\right)^{\beta}+$ $\left[T_{1, I}(\infty)\right]^{-1}$ with four independent fit parameters: $T_{1, I}(0), T_{1, I}(\infty)$, $\tau_{\mathrm{c}}$ and $\beta$. For $\omega_{I} \tau_{\mathrm{c}} \gg 1$ and $T_{1, I \mathrm{fit}}(\omega) \ll T_{1, I}(\infty)$, the function is approximated by a power law with $\xi=2 \beta$. The fitted curves are plotted as solid lines in Fig. $4 \mathrm{a}$. The parameter $\tau_{\mathrm{c}}$ for alkanes in alumina is determined from the relaxation behavior below $50 \mathrm{~Hz}$, where $T_{1, I}\left(\omega_{I}\right)$ changes from a power-law frequency dependence to a constant, i.e., towards a plateau at $T_{1, I}(0)$. Using the analysis presented earlier, this indicates a maximum correlation time $\left(\tau_{c}=\tau_{c, \max }\right)$ of around $20-30 \mathrm{~ms}$, which is at least two orders of magnitude longer than the maximum correlation time of more polar molecules in porous confinement, such as water. Relative to octane, the plateau for decane extends to a higher Larmor frequency, indicating a shorter $\tau_{c \text {,max }}$, despite octane having a higher selfdiffusion coefficient as a bulk liquid. However, at this point $\tau_{\mathrm{c}}$ is also of similar magnitude to the longitudinal relaxation time. Under such conditions the assumptions of standard NMR relaxation theoriessuch as the Wangsness-Bloch-Redfield theory-are not strictly justified as valid, in particular the coarse-graining of time ${ }^{35}$, where spin diffusion may be a part of the relaxation mechanism, or set an upper limit for the relaxation rate in the plateau. Whether this is true requires more information on the physical process responsible for spin relaxation.

High-resolution relaxometry. Field instability in traditional NMRD electromagnets is large compared to spectroscopic dispersion from NMR chemical shifts or inter-spin couplings, resulting in severe or even complete overlap of the signals from nuclei in different chemical groups or different compounds in a mixture. Although additional strategies may prove helpful to assign relaxation rates to distinct chemical groups, (e.g. selective deuteration or other isotopic substitution, inverse Laplace transforms), higher-resolution signal detection would be a more general and direct solution.

Here we illustrate simultaneous measurement and independent fitting of relaxation rates for two chemically distinct ${ }^{1} \mathrm{H}$ environments in methanol $\left(\mathrm{CH}_{3} \mathrm{OH}\right)$. A scalar coupling $\left({ }^{1} J_{\mathrm{CH}}=\right.$ $140.1 \mathrm{~Hz}$ ) between ${ }^{13} \mathrm{C}$ and ${ }^{1} \mathrm{H}$ nuclei in the ${ }^{13} \mathrm{CH}_{3}$ group shifts the corresponding NMR signal by around $\pm 0.5 J_{\mathrm{CH}}$ relative to that of the non-coupled $\mathrm{OH}$, when measured at fields $\left|B_{z, \mathrm{I}}\right| \gg \mid 2 \pi^{1} J_{\mathrm{CH}} /$ $\left(\gamma_{\mathrm{H}}-\gamma_{\mathrm{C}}\right) \mid$. The latter criterion defines the well-known weak heteronuclear coupling regime. Shifts by other multiples of ${ }^{1} J_{\mathrm{CH}}$ between 1 and 2 occur at lower fields. Experimental spectra and simulated positions of the NMR peaks are shown in Fig. 5. Line widths are on the order of $1 \mathrm{~Hz}$, which should also allow spectral resolution of the $\mathrm{CH}_{3}$ groups in methanol, acetone $\left(\mathrm{CH}_{3} \mathrm{COCH}_{3}\right.$, $\left.{ }^{1} J_{\mathrm{CH}}=127 \mathrm{~Hz}\right)$, acetic acid $\left(\mathrm{CH}_{3} \mathrm{COOH}, \quad{ }^{1} J_{\mathrm{CH}}=130 \mathrm{~Hz}\right)$ dimethylsulfoxide $\left(\mathrm{CH}_{3} \mathrm{SOCH}_{3},{ }^{1} J_{\mathrm{CH}}=137 \mathrm{~Hz}\right)$ and other solvents. Isotopomers splittings that arise for couplings over more than one chemical bond, e.g. ${ }^{13} \mathrm{C}^{12} \mathrm{CH}_{3}$, for which ${ }^{2} J_{\mathrm{CH}}=5-30$ $\mathrm{Hz}$, would also be resolvable.

By using the sequence shown in Fig. $3 \mathrm{a}$ to provide a series of

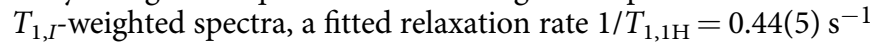
(not plotted) is obtained for the $\mathrm{OH}$ subsystem. Within error, the value does not depend on field. Relaxation rates for the $\mathrm{CH}_{3}$ subsystem are also field independent in the weak-coupling regime above $B_{z, \mathrm{I}}=10 \mu \mathrm{T}$, and are very close to those of $\mathrm{OH}: 1 / T_{1,1 \mathrm{H}}=$ $0.45(14) \mathrm{s}^{-1}$ (not plotted). Both sets of rates refer to relaxation of 


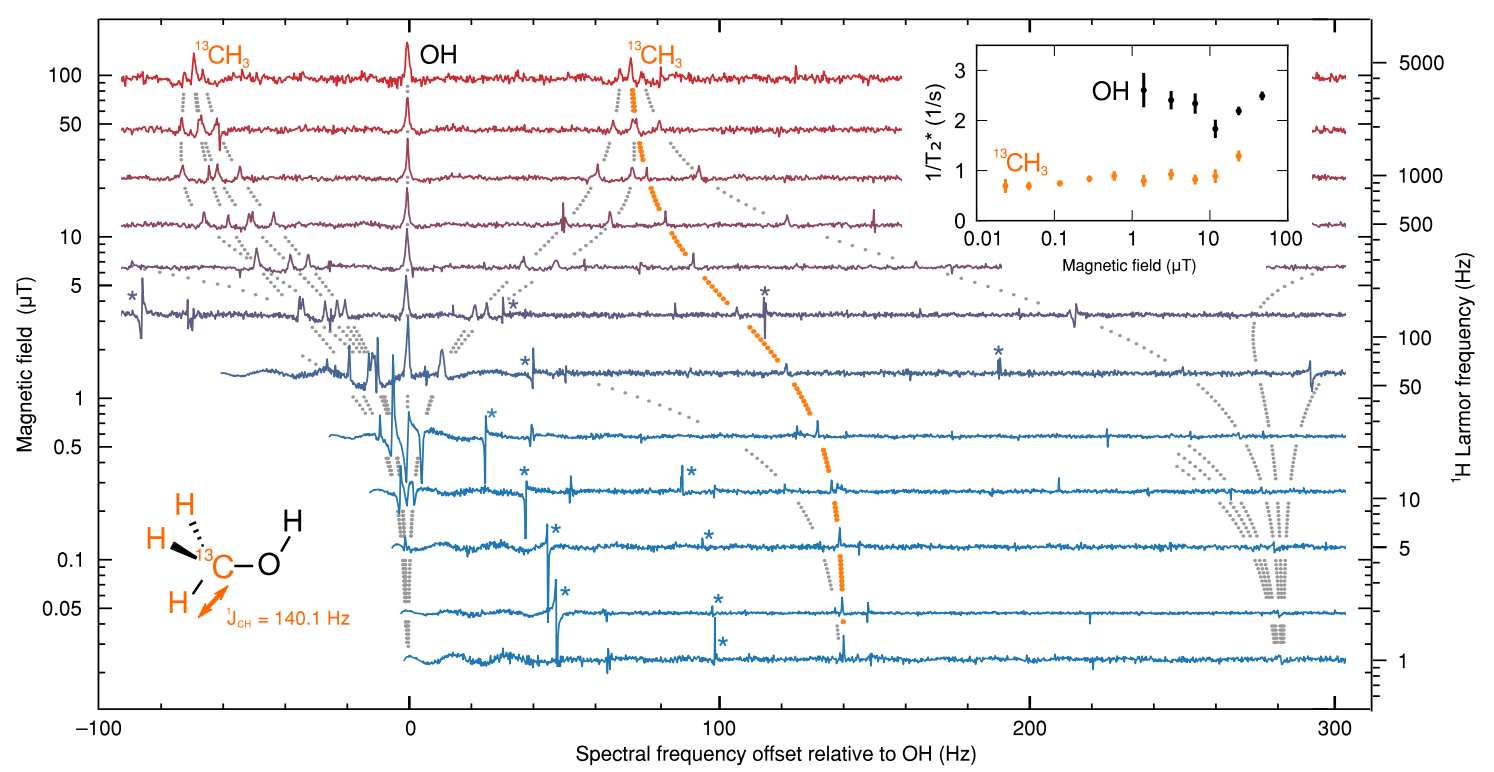

Fig. 5 NMR spectra of ${ }^{13} \mathrm{CH}_{3} \mathrm{OH}$ versus field strength in the range $\mathbf{2 5} \mathbf{n T}-\mathbf{1 0 0} \boldsymbol{\mu}$ T. Horizontal lines show NMR spectra acquired as in Fig. 2, offset vertically by the field at which they were acquired (left scale), and horizontally so as to place the uncoupled $\mathrm{OH}$ resonance at zero. Individual resonances for the chemically distinct $\mathrm{CH}_{3}$ and $\mathrm{OH}$ spin groups are clearly visible and demonstrate relative frequency shifts due to the transition between strong and weak heteronuclear coupling regimes corresponding to low and high field, respectively. The different peaks correspond to different spin combinations; in high field the peaks correspond to single-spin transitions of ${ }^{1} \mathrm{H}$, while at zero field peaks at ${ }^{1} \mathrm{~J}_{\mathrm{CH}}=140.1 \mathrm{~Hz}$ and $2 \times{ }^{1} J_{\mathrm{CH}}=280.2 \mathrm{~Hz}$ correspond,

respectively, to singlet-to-triplet and triplet-to-quintet transitions of the ${ }^{13} \mathrm{CH}_{3}$. Features marked with an asterisk ( ${ }^{\star}$ ), e.g. $50 \mathrm{nT}$ and $\approx 48 \mathrm{~Hz}+\approx 98 \mathrm{~Hz}$, are artefacts due to $50 \mathrm{~Hz}$ line noise and harmonics. Dotted curves show the predicted resonance frequencies as a result of the heteronuclear coupling. Inset shows transverse decay rate $1 / T_{2, l}^{*}$ for the $\mathrm{CH}_{3}$ signal peak (dotted orange curve) and $\mathrm{OH}$ signal peak (at $\mathrm{OHz}$ ) versus field strength. Error bars represent estimated standard deviation errors obtained from least-squares regression analysis.

the ${ }^{1} \mathrm{H}$ spin species and are thus comparable with results obtained via conventional field cycling NMRD.

At lower fields, however, L-S type effects of the scalar coupling between ${ }^{13} \mathrm{C}$ and ${ }^{1} \mathrm{H}_{3}$ lead to a significant contrast in transverse relaxation rates. Of most interest is the peak tending to frequency ${ }^{1} J_{\mathrm{CH}}$ at zero field (orange curve in Fig. 5), which corresponds to singlet-to-triplet coherence in the isolated manifold formed between ${ }^{13} \mathrm{C}$ and the ${ }^{1} \mathrm{H}_{3}$ state of total spin quantum number $1 / 2$. Here the transverse relaxation rate is around 2.5-3 times slower than for the non-coupled OH (Fig. 5, inset). The ${ }^{13} \mathrm{CH}_{3}$ system, therefore, exhibits a type of long-lived spin order in the NMR ensemble ${ }^{47}$. Despite the presence of ${ }^{1} \mathrm{H}-{ }^{1} \mathrm{H}$ and ${ }^{13} \mathrm{C}-{ }^{1} \mathrm{H}$ dipole-dipole couplings, this singlet-to-triplet coherence is long-lived because it is less sensitive to relaxation by fields that are correlated across the ${ }^{13} \mathrm{C}$ and ${ }^{1} \mathrm{H}$ spin groups. This includes much of the intra- ${ }^{13} \mathrm{CH}_{3}$ dipole coupling as well as longer-range couplings. The result has potential importance in applications to probe dipole-dipole interactions at short distances away from the $\mathrm{CH}_{3}$, including intermolecular interactions.

\section{Discussion}

The study demonstrates that unique, important information about nuclear spin relaxation in liquids can be obtained by fast field switching and tunable NMR detection at ultralow magnetic fields.

A basic advantage of the low-field NMR detection is that it eliminates many concerns about magnetic field homogeneity and stability, since magnetic fields are accurately and precisely controlled. This is shown by the result $T_{2} \sim T_{1}$ for the series of TEMPOL solutions. Additionally, as shown for methanol, the Fourier-transform NMR spectrum line width is adequate to resolve spin-spin couplings (even in the alumina system where line widths exceed, $10 \mathrm{~Hz}$ ) and therefore components of liquid mixtures. Ultralow-field NMRD may therefore be able to probe other interplay, such as competitive adsorption between molecules and pore. Besides nonuniformity of applied magnetic fields, conventional NMR is also confounded by sample heterogeneity, especially in multi-phase samples with internal magnetic susceptibility variation, including porous materials, or metal regions. The $\tau_{c}$ values obtained here for alkanes in porous alumina are extremely long by FFC-NMR standards-comparable $\tau_{\mathrm{c}} \mathrm{s}$ are typically probed in high field by pulsed-field gradient (PFG) diffusometry and rotating-frame $\left(T_{1 \rho, \mathrm{I}}\right)$ relaxometry techniques ${ }^{48}$. In most if not all applications, both of the latter are highly susceptible to contamination by poor field homogeneity and radiofrequency offset errors.

Compared to high-field inductive-detected NMRD, ultralowfield OPM-detected NMRD currently has some limitations. A main limitation, resulting from the Hartmann-Hahn matching condition, is that the OPM Faraday rotation signal contains freeprecession responses of the sensor atom and NMR sample spins at the same frequency. The atomic response is at least two orders of magnitude stronger than the NMR signal and can easily saturate the digitizer, which leads to a "dead time" on the order of the optical pumping time $(10 \mathrm{~ms}$, see Fig. $4 \mathrm{~d})$. This currently hinders applications in chemical systems where molecules interact more strongly, namely liquids in nanopores (e.g. zeolites, shale) and interfaces with hydrogen bonding, where relaxation rates are higher. In principle, Q-switching of the optical pumping beam ${ }^{49}$ is a method to accelerate magnetometer recovery after the magnetic field pulses and reduce the dead time down to the field switching time, well below $1 \mathrm{~ms}$, without compromising sensitivity.

Ultralow-field FFC NMRD may also in the future expand study paths when enriched by nuclear spin hyperpolarization. As shown in Fig. 2c, a few tens of scans result in snr $>20 \mathrm{~dB}$, even though the spins are only prepolarized to around 1 part in $10^{8}$ at the 20 
$\mathrm{mT}$ starting field. Nitroxide radical compounds such as TEMPOL are a source of higher electron spin polarization, around 1 part in $10^{5}$ at $20 \mathrm{mT}$, that can be efficiently transferred to nuclei via the Overhauser effect at both high ${ }^{44}$ and ultralow ${ }^{50}$ magnetic fields. Hyperpolarization via surface-supported paramagnetic species and other spin-transfer catalysts may also be an option to study nuclear polarization buildup near pore surfaces, providing information that may differ from relaxation decay. TEMPOL and other persistent radicals are used to prepare hyperpolarized biochemical probes for clinically relevant in vivo observations of disease via MRI ${ }^{51}$. These systems could profit from a knowledge of signal decay mechanisms at ultralow magnetic fields, for sources of image contrast or to minimize polarization losses before imaging/detection.

\section{Methods}

Sample preparation. All samples studied in this work were contained in disposable glass vials ( $12 \mathrm{~mm}$ o.d., $20 \mathrm{~mm}$ length, $1.8 \mathrm{~mL}$ internal volume, $8-425$ thread) sealed with a silicone septum and finger-tight polypropylene screw cap.

Preparation of TEMPOL samples. A $10 \mathrm{mM}$ stock solution of the radical 4-hydroxy2,2,6,6-tetramethylpiperidin-1-oxyl (Sigma Aldrich, CAS: 2226-96-2) was prepared in $5.0 \mathrm{~mL}$ deoxygenated milli-Q water and diluted to concentrations of $0.5,1,2,3$, 4,5 , and $10 \mathrm{mM}$ with deoxygenated milli-Q water. The diluted solutions were not further de-gassed.

Preparation of porous materials samples. Cylindrical extrudate pellets of mesoporous $\gamma$-alumina (Alfa Aesar product 43855, lot Y04D039: $3 \mathrm{~mm}$ diameter, $3 \mathrm{~mm}$ length, $9 \mathrm{~nm} \mathrm{BJH}$ mean pore diameter, Langmuir surface area $250 \mathrm{~m}^{2} \mathrm{~g}^{-1}$ ) and anatase titania (Alfa Aesar product 44429, lot Z05D026: $3 \mathrm{~mm}$ diameter, $4 \mathrm{~mm}$ length, 7-10 nm mean pore size, Langmuir surface area $150 \mathrm{~m}^{2} \mathrm{~g}^{-1}$ ) were obtained commercially. Pellets were oven-dried at $120^{\circ} \mathrm{C}$ for $12 \mathrm{~h}$ to remove physisorbed $\mathrm{H}_{2} \mathrm{O}$ and then imbibed in neat $\mathrm{n}$-alkane for at least $12 \mathrm{~h}$ after recording the dry mass. Excess liquid on the pellet outer surface was gently removed using tissue paper. The pellets were then placed in a vial (see Fig. 4a), sealed with the cap and the combined mass of pellet and imbibed hydrocarbon was recorded.

Preparation of methanol sample. $0.9 \mathrm{~g}$ of ${ }^{13} \mathrm{C}$-methanol $\left({ }^{13} \mathrm{CH}_{3} \mathrm{OH} 99 \%\right.$, Sigma Aldrich product 277177) was added to the sample vial without dilution, then followed by $\mathrm{N}_{2}$ bubbling (2-3 min) to displace dissolved paramagnetic $\mathrm{O}_{2}$.

Optical magnetometer. The magnetometer used to detect ${ }^{1} \mathrm{H}$ precession signals in the NMR samples was operated as follows. A cuboid borosilicate glass cell of inner dimensions $5 \times 5 \times 8 \mathrm{~mm}^{3}$ contained a droplet of rubidium- 87 metal and $90 \mathrm{kPa} \mathrm{N}_{2}$ buffer gas (Twinleaf LLC). The cell was electrically heated to $150^{\circ} \mathrm{C}$ to vaporize the alkali metal. A circularly polarized light beam along $z$-axis $(3 \mathrm{~mW}$, tuned to the center of the collision-shifted $D_{1}$ wavelength) optically pumped the atomic spin polarization to $S_{z} \approx 0.5$. Faraday rotation in a second, linearly polarized light beam $(10 \mathrm{~mW}, 65 \mathrm{GHz}$ red-shifted from the pump, along $x$-axis) was used to nonresonantly probe the $S_{x}$ component of atomic polarization. The probe beam was linearly polarized and slightly detuned from the ${ }^{87} \mathrm{Rb} \mathrm{D}_{1}$ transition, such that on passing through the cell along the $x$-axis its axis of polarization was optically rotated by an angle proportional to $S_{x}$. The Faraday rotation was detected by polarimetry using a differential photodetector (Thorlabs PDB210A), which produced an analog voltage signal that was conditioned (amplified, filtered to eliminate high-frequency noise and dc offset) and digitized (60 ksps, 16-bit $\pm 5 \mathrm{~V}$ ADC range) before storage and further processing on a computer.

Magnetic coils and shielding. The vapor cell and heating assembly was placed as close as possible to the NMR sample at a standoff distance $d_{1}=3.5 \mathrm{~mm}$ between outer walls of the vial and atomic vapor cell (see Fig. 2a). In order of increasing distance away from the NMR sample, $d_{1}$ accounts for (i) S2 + S3 coil windings (34 AWG enameled copper wire, solenoid length $13 \mathrm{~cm}$, diameter $14 \mathrm{~mm}$ ), (ii) a carbon-fiber support structure, (iii) S1 coil windings (36 AWG enameled copper wire, solenoid length $2.5 \mathrm{~cm}$, single layer), (iv) a water-cooling jacket (de-ionized water, flow rate $1 \mathrm{~mL} \mathrm{~s}^{-1}$ ) to remove heat deposited when the polarizing coil is energized and to maintain a stable sample temperature, (v) a PEEK support structure, and (vi) an air gap for further thermal insulation. The entire structure was operated within a cylindrical magnetic shield (Twinleaf LLC, model MS-1F) of $20 \mathrm{~cm}$ outer diameter and length $30 \mathrm{~cm}$. The main axis of the cylinder was co-axial with the pump beam axis.

Field-to-current ratios inside each coil were calibrated using the frequency of ${ }^{1} \mathrm{H}$ precession in de-ionized water. These were $\mathrm{S} 2: 7.59(3) \mu \mathrm{T} \mathrm{mA}{ }^{-1}$; S3: 7.50(3) $\mu \mathrm{T}$ $\mathrm{mA}^{-1}$; S4: 150.1(5) nT mA ${ }^{-1}$. The atomic spin precession frequency at the atomic vapor cell was used to calibrate the external field of coils S2: $-11.1(3) \mathrm{nT} \mathrm{mA}^{-1}$ and S3: $-4.4(2) \mathrm{nT} \mathrm{mA}^{-1}$, which equate to 1460 and $580 \mathrm{ppm}$ of the field at the vial, respectively.

Magnetic noise spectra. An ac test signal of $\pm 8 \mathrm{pT}$ along the $y$-axis was applied to calibrate the Faraday response as a function of frequency and $z$ bias field. The calibration vs. frequency was used to scale the spectral response of the balanced photodetector from units of $V / \sqrt{\mathrm{Hz}}$ into $T / \sqrt{\mathrm{Hz}}$. The maximum magnetic response at a given bias field was confirmed to equal the tuning condition where the atomic Larmor frequency matched the frequency of the ac signal, given the prior calibration of the magnetic field at the sample vial and magnetometer cell.

Field switching. Timing of the NMR pulse sequences and data acquisition were controlled by a microcontroller (Kinetis K20 series: time base $2 \mu$ s, precision $17 \mathrm{~ns}$, $\mathrm{CPU}$ speed $120 \mathrm{MHz}$ ). Current to the polarizing coil was switched via a dual $\mathrm{H}$ bridge circuit with parallel flyback diodes and the switching time was $<1.0 \mathrm{~ms}$. Coils S2 and S4 were connected to a low-noise precision current source (Twinleaf model CSB-10, 20-bit resolution over $\pm 10 \mathrm{~mA}$ ) with a low-pass LC filter in series, resulting in a combined switching and settling time of order $100 \mathrm{~ms}$. The FFC solenoid coil S3 operated at a current $<1 \mathrm{~mA}$ direct from the microcontroller digital-to-analog converter (12-bit resolution, 0-1 mA) for rapid and precise field switching without feedback controls. Typical S3 switching times were $0.25 \mathrm{~ms}$ and the accuracy (determined from standard error in the mean NMR center frequency over repeated scans at $\omega_{I} /(2 \pi)=550 \mathrm{~Hz}$, see Fig. $4 \mathrm{~b}$ inset) was around $1 \mathrm{nT}$. The residual interior field of the MuMetal shield along the $x, y$, and $z$ axes of $\sim 10 \mathrm{nT}$ was also compensated for.

Under steady-state conditions with the pre-polarizing coil turned off, the cooling system maintained a temperature of $27-28^{\circ} \mathrm{C}$ at a thermocouple attached to the outside wall of the sample vial. The steady-state temperature rose to $30-31{ }^{\circ} \mathrm{C}$ when the polarizing coil was energized at $20 \mathrm{mT}(2.2 \mathrm{~A})$.

\section{Data availability}

The raw data generated in this study have been deposited in the OpenAIRE database under accession code https://doi.org/10.5281/zenodo.4840653.

Received: 25 November 2020; Accepted: 7 June 2021; Published online: 30 June 2021

\section{References}

1. Kimmich, R. Field cycling in NMR relaxation spectroscopy applications in biological, chemical and polymer physics. Bull. Magn. Reson. 1, 195-218 (1979).

2. Kimmich, R. (Ed.), Field Cycling NMR Relaxometry: Instrumentation, Model Theories and Applications (The Royal Society of Chemistry, Oxford, 2018).

3. Kimmich, R. \& Anoardo, E. Field-cycling NMR relaxometry. Progr. Nucl. Magn. Reson. Spectrosc. 44, 257-320 (2004).

4. Schneider, D. J. \& Freed, J. H. Spin relaxation and motional dynamics. In Advances in Chemical Physics (eds. Hirschfelder, J.O., Wyatt, R.E. and Coalson, R.D.) 387-527 (John Wiley \& Sons, Ltd., 2007).

5. Deutch, J. M. \& Oppenheim, I. Time correlation functions in nuclear magnetic relaxation. Adv. Opt. Magn. Reson. 3, 43-78 (1968).

6. Bychuk, O. V. \& O'Shaughnessy, B. Anomalous diffusion at liquid surfaces. Phys. Rev. Lett. 74, 1795-1798 (1995).

7. Guo, J.-C. Advances in low-field nuclear magnetic resonance (NMR) technologies applied for characterization of pore space inside rocks: a critical review. Petr. Sci. 17, 1281-1297 (2020).

8. Waddington, D. E. J., Boele, T., Maschmeyer, R., Kuncic, Z. \& Rosen, M. S. High-sensitivity in vivo contrast for ultra-low field magnetic resonance imaging using superparamagnetic iron oxide nanoparticles. Sci. Adv. 6, eabb0998 (2020).

9. Korb, J.-P. Multiscale nuclear magnetic relaxation dispersion of complex liquids in bulk and confinement. Progr. Nucl. Magn. Reson. Spectrosc. 104 12-55 (2018)

10. Job, C., Zajicek, J. \& Brown, M. F. Fast field-cycling nuclear magnetic resonance spectrometer. Rev. Sci. Instrum. 67, 2113-2122 (1996).

11. Ward-Williams, J., Korb, J.-P. \& Gladden, L. F. Insights into functionalityspecific adsorption dynamics and stable reaction intermediates using fast field cycling NMR. J. Phys. Chem. C 122, 20271-20278 (2018).

12. Ferrante, G. \& Sykora, S. Technical aspects of fast field cycling. Adv. Inorg. Chem. 57, 405-470 (2005).

13. Anoardo, E., Galli, G. \& Ferrante, G. Fast-field-cycling NMR: applications and instrumentation. Appl. Magn. Reson. 20, 365-404 (2001).

14. Anoardo, E. \& Ferrante, G. M. Magnetic field compensation for field-cycling NMR relaxometry in the ULF band. Appl. Magn. Reson. 24, 85 (2003).

15. Kresse, B., Privalov, A. F. \& Fujara, F. NMR field-cycling at ultralow magnetic fields. Solid State Nucl. Magn. Reson. 40, 1926-2040 (2011). 
16. Chou, C.-Y., Chu, M., Chang, C. F. \& Huang, T. A compact high-speed mechanical sample shuttle for field-dependent high-resolution solution NMR. J. Magn. Reson. 214, 302-308 (2012).

17. Kaseman, D. C. Design and implementation of a J-coupled spectrometer for multidimensional structure and relaxation detection at low magnetic fields. Rev. Sci. Instrum. 91, 054103 (2020).

18. Ganssle, P. J. Ultra-low-field NMR relaxation and diffusion measurements using an optical magnetometer. Angew. Chem. Int. Ed. 53, 9766-9770 (2014).

19. Tayler, M. C. D., Ward-Williams, J. \& Gladden, L. F. NMR relaxation in porous materials at zero and ultralow magnetic fields. J. Magn. Reson. 297, 1-8 (2018).

20. Zhukov, I. V. Field-cycling NMR experiments in an ultra-wide magnetic field range: relaxation and coherent polarization transfer. Phys. Chem. Chem. Phys. 20, 12396-12405 (2018)

21. Savukov, I. M. \& Romalis, M. V. NMR detection with an atomic magnetometer. Phys. Rev. Lett. 94, 123001 (2005).

22. Savukov, I. M., Seltzer, S. J. \& Romalis, M. V. Detection of NMR signals with a radio-frequency atomic magnetometer. J. Magn. Reson. 185, 214-220 (2007).

23. Blanchard, J. W. \& Budker, D. Zero- to ultralow-field NMR. eMagRes 5, 1395-1409 (2016).

24. Tayler, M. C. D. Invited review article: Instrumentation for nuclear magnetic resonance in zero and ultralow magnetic fields. Rev. Sci. Instrum. 88, 091101 (2017).

25. Happer, W. \& Tam, A. C. Effect of rapid spin exchange on the magnetic resonance spectrum of alkali vapors. Phys. Rev. A 16, 1877-1891 (1977).

26. Savukov, I. M. \& Romalis, M. V. Effects of spin-exchange collisions in a highdensity alkali-metal vapor in low magnetic fields. Phys. Rev. A 71, 023405 (2005).

27. Allred, J. C., Lyman, R. N., Kornack, T. W. \& Romalis, M. V. High-sensitivity atomic magnetometer unaffected by spin-exchange relaxation. Phys. Rev. Lett. 89, 130801 (2002).

28. Ledbetter, M. P., Savukov, I. M., Acosta, V. M., Budker, D. \& Romalis, M. V. Spin-exchange-relaxation-free magnetometry with Cs vapor. Phys. Rev. A 77, 033408 (2008).

29. Budker, D. \& Romalis, M. V. Optical magnetometry. Nat. Phys. 3, 227-234 (2007).

30. Budker, D. \& Jackson Kimball, D. F. (Eds.) Optical Magnetometry (Cambridge University Press, 2013).

31. McDermott, R. et al. Liquid-state NMR and scalar couplings in micro-tesla magnetic fields. Science 295, 2247-2249 (2002).

32. Trahms, L. \& Burghoff, M. NMR at very low fields. Magn. Reson. Imaging 28, 1244-1250 (2010).

33. Appelt, S., Kühn, H., Häsing, F. \& Blümich, B. Chemical analysis by ultrahighresolution nuclear magnetic resonance in the Earth's magnetic field. Nat. Phys. 2, 105-109 (2006)

34. Suefke, M., Liebisch, A., Blümich, B. \& Appelt, S. External high-quality-factor resonator tunes up nuclear magnetic resonance. Nat. Phys. 11, 767-771 (2015).

35. Redfield, A. G. The theory of relaxation processes. Adv. Magn. Opt. Reson. 1, 1-32 (1965).

36. Kowalewski, J. \& Maler, L. Nuclear Spin Relaxation in Liquids (CRC Press, Boca Raton, 2018)

37. Halle, B., Jóhannesson, H. \& Venu, K. Model-free analysis of stretched relaxation dispersions. J. Magn. Reson. 135, 1-13 (1998).

38. Klafter, J. \& Schlesinger, M. F. On the relationship among three theories of relaxation in disordered systems. Proc. Natl. Acad. Sci USA 83, 848-851 (1986).

39. McDonald, P. J. \& Faux, D. A. Nuclear-magnetic-resonance relaxation rates for fluid confined to closed, channel or planar pores. Phys. Rev. E 98, 063110 (2018).

40. Miyaguchi, T., Uneyama, T. \& Akimoto, T. Brownian motion with alternately fluctuating diffusivity: stretched-exponential and power-law relaxation. Phys. Rev. E 100, 012116 (2019).

41. Kimmich, R. Strange kinetics, porous materials and NMR. Chem. Phys. 284, 253-285 (2002).

42. Zavada, T. \& Kimmich, R. Surface fractals probed by adsorbate spin-lattice relaxation dispersion. Phys. Rev. E 59, 5848-5854 (1999).

43. Lewandowski, M. \& Gwozdinski, K. Nitroxides as antioxidants and anticancer drugs. Int. J. Mol. Sci. 18, 2490 (2017).

44. Prandolini, M. J., Denysenkov, V. P., Gafurov, M., Endeward, B. \& Prisner, T. F. High-field dynamic nuclear polarization in aqueous solutions. J. Am. Chem. Soc. 131, 6090-6092 (2009).
45. Neugebauer, P. Liquid state DNP of water at $9.2 \mathrm{~T}$ : an experimental access to saturation. Phys. Chem. Chem. Phys. 15, 6049-6056 (2013).

46. Ward-Williams, J., Korb, J.-P., Rozing, L., Sederman, A. J., Mantle, M. D. \& Gladden, L. F. Characterizing solid-liquid interactions in a mesoporous catalyst support using variable-temperature fast field cycling NMR. J. Phys. Chem. C 125, 8767-8778 (2021).

47. Pileio, G. (Ed.), Long-lived Nuclear Spin Order: Theory and Applications (Chapters 20 and 23), volume 22 of New Developments in NMR, Royal Society of Chemistry (2020)

48. Price, W. S. NMR Studies of Translational Motion (Cambridge University Press, 2009)

49. Limes, M. E. Portable magnetometry for detection of biomagnetism in ambient environments. Phys. Rev. Appl. 14, 011002 (2020).

50. Hilschenz, I. Dynamic nuclear polarisation of liquids at one microtesla using circularly polarised RF with application to millimetre resolution MRI. J. Magn. Reson. 305, 138-145 (2019).

51. Sriram, R., Kurhanewicz, J. \& Vigneron, D. B. Hyperpolarized carbon-13 MRI and MRS studies. eMagRes 3, 311-324 (2014)

\section{Acknowledgements}

The work described is funded by: EU H2020 Marie Skłodowska-Curie Actions project ITN ZULF-NMR (Grant Agreement No. 766402); Spanish MINECO projects OCARINA (Grant No. PGC2018-097056-B-I00), the Severo Ochoa program (Grant No. SEV-2015-0522); Generalitat de Catalunya through the CERCA program; Agència de Gestió d'Ajuts Universitaris i de Recerca Grant No. 2017-SGR-1354; Secretaria d'Universitats i Recerca del Departament d'Empresa i Coneixement de la Generalitat de Catalunya, co-funded by the European Union Regional Development Fund within the ERDF Operational Program of Catalunya (project QuantumCat, ref. 001-P-001644); Fundació Privada Cellex; Fundació Mir-Puig; MCD Tayler acknowledges financial support through the Junior Leader Postdoctoral Fellowship Program from "La Caixa" Banking Foundation (project LCF/BQ/PI19/ 11690021). The authors also thank Jordan Ward-Williams and Lynn Gladden (University of Cambridge) for providing samples of porous alumina and titania, and for discussions.

\section{Author contributions}

M.C.D.T. proposed the study. S.B. prepared the samples, measured and analyzed the experimental data and together with M.C.D.T. built the experimental apparatus and made the theoretical interpretation. M.C.D.T. wrote the manuscript with input from al authors. All authors reviewed the manuscript and suggested improvements. M.C.D.T. and M.W.M. supervised the overall research effort.

\section{Competing interests}

The authors declare no competing interests.

\section{Additional information}

Correspondence and requests for materials should be addressed to M.C.D.T.

Peer review information Nature Communications thanks the anonymous reviewers for their contribution to the peer review of this work.

Reprints and permission information is available at http://www.nature.com/reprints

Publisher's note Springer Nature remains neutral with regard to jurisdictional claims in published maps and institutional affiliations.

Open Access This article is licensed under a Creative Commons Attribution 4.0 International License, which permits use, sharing, adaptation, distribution and reproduction in any medium or format, as long as you give appropriate credit to the original author(s) and the source, provide a link to the Creative Commons license, and indicate if changes were made. The images or other third party material in this article are included in the article's Creative Commons license, unless indicated otherwise in a credit line to the material. If material is not included in the article's Creative Commons license and your intended use is not permitted by statutory regulation or exceeds the permitted use, you will need to obtain permission directly from the copyright holder. To view a copy of this license, visit http://creativecommons.org/ licenses/by/4.0/.

(C) The Author(s) 2021 